\title{
Evaluating the Therapeutic Viability of Bacterially Expressed Human TLK1B-Kinase Domain for Cancer Drug Design
}

\section{Delna Johnson}

Indian Institute of Technology Gandhinagar

Javeena Hussain

Indian Institute of Technology Gandhinagar

Siddhant Bhoir

Indian Institute of Technology Gandhinagar

Parul Sahsrawat

Indian Institute of Technology Gandhinagar

Tanya Hans

Indian Institute of Technology Gandhinagar

C Vaishali

Indian Institute of Technology Gandhinagar

Vijay Thiruvenkatam

Indian Institute of Technology Gandhinagar

Sivapriya Kirubakaran ( $\sim$ priyak@iitgn.ac.in )

Indian Institute of Technology Gandhinagar

\section{Research Article}

Keywords:

Posted Date: February 21st, 2022

DOI: https://doi.org/10.21203/rs.3.rs-1314176/v1

License: (1) This work is licensed under a Creative Commons Attribution 4.0 International License. Read Full License 


\section{Abstract}

The role of protein kinases is vital in diverse cellular functions. The alterations in the expression patterns of protein kinases often implicate human cancer initiation and progression. Human Tousled-like kinases (TLKs) are evolutionary kinases found in the cell signalling pathway and are involved in DNA repair, replication and chromosomal integrity. The direct association of TLKs to cancer; amplification of both $T L K 1 / 1 B$ and $T L K 2$ has made them viable molecular targets for anticancer therapy. Several reports demonstrate numerous functions of TLKs in development of disease via different interacting partners. However, a detailed understanding of its substrates and regulation has yet remained elusive. In this report, through preliminary biophysical and biochemical characterization, we investigate and determine the usability of the recombinant Human Tousled-like Kinase 1B-Kinase Domain (hTLK1B-KD) purified from Escherichia coli for structural and functional studies. By illustrating hTLK1B-KD as an example, our attempts to generate a stable, homogenously dephosphorylated, and catalytically active hTLK1B-KD in high yields is utilizing a bacteriophage $\lambda$ protein phosphatase (LPP) coexpression system represents a fundamental step towards the structure-based design of TLK-specific inhibitors.

\section{Introduction}

The cell signalling pathways are often regulated by kinases ${ }^{1}$. The perturbation to these pathways because of kinase dysfunction caused due to mutations, translocations, and upregulation events often results in unanticipated detrimental consequences such as diabetes, inflammation, and mainly cancer ${ }^{2}$. Cancer is a primary health concern worldwide ${ }^{3}$. According to the Cancer Facts \& Figures of American Cancer Society, the number of new cancer cases was expected to be approximately 1.9 million in the United States (US) in 2021. However, intriguingly, the Cancer Statistics 2021 reports the decline of death rate from cancer in the US consistently by $31 \%$ over the past two decades. This drop in the cancer mortality rate is predominantly due to a reduction in smoking and advancement in the disease diagnosis and treatment ${ }^{4}$. With the success of the discovery of the imatinib, the first kinase inhibitor, a multitude of the current drug development efforts considerably focuses on targeting aberrant kinases with the selective inhibitors, thus, uncovering their tremendous treatment potential for cancer and other diseases ${ }^{3}$,

${ }^{5}$. Nevertheless, many factors, such as tumour microenvironment, specific tumour genetics, pharmacogenomics, and drug resistance puzzle the clinical efficacy of these molecules and present remarkable challenges towards kinase drug discovery ${ }^{6}$. Until now, the overall focus of kinase drug development and clinical efforts has been exclusive to oncology, with only one JAK inhibitor tofacitinib for the non-oncology applications of arthritis ${ }^{3,7}$. Owing to the challenges, there are only a few FDA approved kinase inhibitors available for the treatment of breast and lung cancer, after the first protein kinase inhibitor in $2001^{8,9}$. Hence, there is a compelling need to devise improved methods to modulate the kinase function. The kinases were authenticated as important molecular targets by the development of leading edge chemical biology techniques. In 1991, the three dimensional crystal stricture of a Protein Kinase-A domain provided a fundamental model for the inhibitor design. In the following years, the number of human catalytic domain model structures increased expeditiously to over more than 200, thus, 
emphasizing the role of structural biology in understanding the translational aspects of this strikingly dynamic protein family ${ }^{10}$. Notably, the high-resolution crystal structure analysis of the selective inhibitorprotein complexes offer explanations for the inhibitor selectivity and identify their unique molecular mechanisms, hence, providing an excellent opportunity in developing a kinase target area. It also provides the basis for optimism that we might overcome the problems with the existing antikinase therapies ${ }^{10,11}$. Precisely, the catalytic domains are the major sites involved in the kinetic reactions and are highly targeted for kinase inhibitor therapy. It is pertinent to overproduce soluble kinase domains in comparison to their full-length counterparts. Since the kinase domain can easily be engineered and expressed in high yield, the development of selective inhibitors would also be fostered, thereby offering an advantage over currently available high throughput platforms ${ }^{12-14}$. All the 518 protein kinases encoded by a human genome share a highly conserved catalytic domain in regards to their sequence and structure. However, they mainly differ in their regulation. The major catalytic site is the ATP-binding pocket, called as the hinge region, that is located between the $\mathrm{N}$-terminal and $\mathrm{C}$-terminal lobes ${ }^{10,11}$. This catalytic site is highly explored especially for targeted drug design to achieve selectivity and to understand the molecular structure of the kinase ${ }^{10}$. The determination of kinase domain structures by employing high-resolution $\mathrm{X}$ ray crystallography requires a protein to be produced in high yields ${ }^{15}$. The human kinase expression achieved in insect cells is substantial ${ }^{16,17}$. Although successful, this method suffers from high experimental costs and is time demanding. Alternatively, the recombinant bacterial expression system offers comparable performance advantages over insect cell culture, including shorter generation times, low costs and increased protein yields. While the removal of the regulatory and other auxiliary regions can adversely affect the expression of the catalytic domains, coexpression along with a protein phosphatase from bacteriophage $\lambda$ is shown to enhance their expression in a soluble and homogeneously dephosphorylated form greatly ${ }^{18,19}$. In our previously published report, we could successfully demonstrate the soluble and homogeneously dephosphorylated expression and purification of wild-type, full-length Human Tousled Like Kinase, (hTLK1B) using such coexpression strategy ${ }^{20}$. Since TLKs are involved in DNA repair mechanism and often upregulated in case of breast or prostate cancer, they are considered to be clinically relevant molecular targets for anticancer therapy ${ }^{21}$. However, surprisingly, the automated screen panel on kinase domain constructs generated by Parton et al. did not report the expression profile of Human TLK1B kinase domain ${ }^{22}$. Hence, we envisaged if this simple, robust and efficient protocol can be extensively applied to the hTLK1B kinase domain (hTLK1B-KD) construct as well.

As a first step towards our research goal, we cloned hTLK1B-KD into a pETDUET-1 vector harbouring a coexpression capacity of two target genes. Impressively, we could obtain ample amounts ( $10 \mathrm{mg}$ per litre of the bacterial culture) of the homogeneously dephosphorylated and soluble, form of biologically active hTLK1B-KD with exceptional purity in a single purification step via 6 -polyhistidine tags, which can then be used for structural, functional and in vitro drug screening studies. Our protocol provides a significant improvement upon the three-step approach described for purification of hTLK2-KD ${ }^{23}$, thus, increasing the protein yields together with managing time, costs and resources. We also evaluated the 
therapeutic viability of the purified hTLK1B through inhibition studies and identified two potent in-house synthesised compounds. These second generation inhibitors were synthesised to complete the structureactivity relationship studies of phenothiazine analogue with respect to TLKs, as our previously reported inhibitor $\mathrm{J} 54$ had shown interesting results during the in vivo screening ${ }^{24}$. We speculate that our sincere efforts in understanding the kinase domain structure, hTLK1B-KD in this case might help the new design of combination and single-agent therapies to enhance the prospects of mitigating challenges involved in kinase drug discovery.

\section{Results}

\subsection{Cloning, expression, and purification of recombinant 6x-polyhistidine-tagged hTLK1B-KD}

To construct a hTLK1B-KD-LPP-pETDUET-1 expression vector by the conventional PCR-based cloning approach, we took advantage of our previously generated wild-type, full-length hTLK1B-pETDUET-1Lambda Protein Phosphatase plasmid as a template for the PCR amplification ${ }^{20}$ (Supplementary Figure, S1). We successfully employed the cloning of hTLK1B-KD into the multiple cloning site MCS-1 of the pETDUET-1 expression vector harbouring the $6 \mathrm{x}$-polyhistidine tag at the $\mathrm{N}$-terminus, with ampicillin as an antibiotic marker gene (Supplementary Figure, S2). Purposely, we kept the MCS-2 of the same vector unmodified as it already had bacteriophage $\lambda$ protein phosphatase open reading frame (ORF) subcloned into it, which facilitates enhanced solubility and dephosphorylation of the hTLK1B-KD during its expression. The cloned construct was further confirmed by the restriction digestion analysis of the plasmid extracted from the positive white colonies and examined its band patterns (Supplementary Figure, S3). Finally, the recombinant hTLK1B-KD-LPP-pETDUET-1 plasmid was expressed into Escherichia coli Rosetta Gami $2^{\text {tw }}$ (DE3) pLysS cells (Supplementary Figure, S4) supplemented with $50 \mu \mathrm{g} / \mu \mathrm{L}$ ampicillin Luria-Bertani Broth. Almost 50\% of the recombinant hTLK1B-KD is expressed in the soluble form when induced with $0.5 \mathrm{mM} \mathrm{IPTG}$ and expressed at $25^{\circ} \mathrm{C}$ for $9 \mathrm{hrs}$, as observed in (Fig. 1a).

The cell lysate was subjected for purification steps to isolate the soluble fractions, initially using the NiNTA affinity chromatography (Fig. 1b) at a higher imidazole concentration of $200 \mathrm{mM}$. Finally, Size Exclusion Chromatography was carried out as a final polishing step and for buffer exchange to remove any other remaining impurities as well as to avoid aggregation (Supplementary Figure, S7). The recombinant hTLK1B-KD protein used here exemplifies the most desirable outcome based on the generic IMAC purification process. In the case of hTLK1B_KD, a significant improvement in purity and yield is observed just after the single affinity purification step. Our one-step purification strategy yields about $10 \mathrm{mg}$ per litre of the bacterial culture as pure, stable, homogeneously unphosphorylated, well-folded and biologically active hTLK1B-KD protein. The monomeric presence of the purified hTLK1B-KD was confirmed with a molecular mass of $36,760.977 \mathrm{Da}$, as expected from the calculated mass of $36,617.66$ Da (Supplementary Figure, S8). 


\subsection{Determination of biological activity and kinetic parameters of recombinant $6 x$-polyhistidine-tagged hTLK1B_KD}

The enzymatic activity was measured using the ADP-Glo ${ }^{\text {TM }}$ Kinase Assay by quantifying the ADP generated during the kinase reaction. We used this assay to monitor the biological activity of recombinant hTLK1B_KD using ASF1a as a substrate. We titrated the hTLK1B_KD kinase from nanomolar to micromolar concentrations to determine the half-maximal effective concentration $\left(\mathrm{EC}_{50}\right)$ of the enzyme, which was found to be $0.708 \mu \mathrm{M}$ (Fig. 2a). We report that the bacterially expressed hTLK1B$\mathrm{KD}$ is reasonably active in the experimental conditions tested with a $\mathrm{K}_{\text {cat }}$ of $108.5 \mathrm{~min}^{-1}$. Surprisingly, its full-length counterpart, hTLK1B, showed a $\mathrm{K}_{\text {cat }}$ of $0.13 \mathrm{~min}^{-1}$, making it unusually less active ${ }^{20}$. We presume that the removal of the $\mathrm{N}$-terminal domains might have resulted in a kinase domain with high basal activity ${ }^{25}$, which further leads to an interesting question that the $\mathrm{N}$-terminal domain of Human TLKs has a regulatory function too. Human TLKs are known to dimerize and autophosphorylate ${ }^{26}$, and the mechanism is yet inadequately understood.

Therefore, we speculate that before activation, the $\mathrm{N}$-terminal domain binds to the $\mathrm{C}$-terminal kinase domain thereby inhibits the activity of the kinase domain. However, upon phosphorylation, when the Cterminal kinase domain is activated and the interaction between the $\mathrm{N}$-terminal domain and the $\mathrm{C}$ terminal kinase domain is disrupted. The results presented in Fig. $2 \mathrm{~b}$ and $2 \mathrm{c}$ are descriptive of the data generated with the hTLK1B-KD at a broad range of ATP and substrate concentrations to determine the $\mathrm{K}_{\mathrm{m}}$ for ATP and ASF1a, respectively. These results show $\mathrm{K}_{\mathrm{m}}$ of $287.7 \mu \mathrm{M}$ for ATP, which is indicative of the fact that hTLK1B-KD is a high ATP requiring kinase. The $\mathrm{K}_{\mathrm{m}}$ for the ASF1a substrate was determined to be $0.853 \mu \mathrm{M}$. From the individual experiments, the amounts of kinase, substrate, ATP, reaction temperature, and incubation times were optimized for the optimal performance of the assay.

\subsection{Circular dichroism (CD) analysis of recombinant $6 \mathrm{x}-$ polyhistidine-tagged hTLK1B_KD}

We evaluated the data on the complete amino acid sequence computed by PSIPRED analysis (Supplementary Figure, S9) to predict the secondary structure from the sequence. The expected structure contained $73.6 \%$ a-helix, $1.2 \% \beta$-sheets, and remaining $25.2 \%$ random coils. We compared these data with those obtained from the CD spectral analysis of hTLK1B-KD, and we found it to be in a definitive agreement with PSIPRED data. The CD spectrum of purified hTLK1B-KD is shown in Fig. 3 with two separate minima exist at 210 and $221 \mathrm{~nm}$. The secondary structure of the protein was also gauged through the K2D3 software (Figure S9). The a-helix, the $\beta$-sheets and the random coils content differs from its full-length counterpart ${ }^{20}$. Based on the available scientific data and our observations, we cannot wholly overrule the possible role of the significant N-terminal extension (absent from hTLK1B-KD) consisting of putative nuclear localization sequences, the coiled-coiled regions, and the glutamine-rich 
domain in protein structure, dynamics, and function. We speculate that the $\mathrm{N}$-terminus and the intrinsically disordered regions and spacer domains might juxtapose to stable the structure to facilitate protein interactions, and may also alter the stability of catalytic loop segments. However, an in-depth structural study is required to understand the functional role of non-catalytic regions and their unique molecular interactions, which is yet an open question to address.

\subsection{In-house chemically-synthesized phenothiazine-based small-molecule inhibitors for hTLK1B-KD}

To demonstarte the flexibility and usability of recombinant hTLK1B-KD, we subjected the in-house chemically-synthesized phenothiazine based small-molecule inhibitors for in vitro studies. Phenothiazine (PTZs) which are well-known for their biological properties such as antimicrobial ${ }^{27}$, antimalarial ${ }^{28}$, antihelminthic ${ }^{29}$, analgesic ${ }^{30}$ and anti-inflammatory ${ }^{31}$, and recently anticancer ${ }^{32}$. The phenothiazine based TLK1/1B inhibitors [Thioridazine (THD), Promethazine (PMZ), Trifluoperazine (TFPZ), Levomepromazine (LVPZ), and Chlorpromazine Hydrochloride (CPZ)] were first identified by Ronald, S. et al. (2013) ${ }^{33}$ in a fluorescent-based high-throughput screen. THD was found to be the most potent and inhibitory. THD has two alkyls chain linkers with piperidine as a side-chain/terminal amine group and $\mathrm{SCH}_{3}$ that plays an essential role in the D2 receptor binding. Reports suggestsC2-unsubstituted PTZs lack antipsychotic properties due to inadequate dopaminergic receptor 2-binding activity and also exhibit neuroprotective effects through their anti-inflammatory and potent free radical scavenging properties ${ }^{34}$, 35. Moreover, our previously synthesized molecule J54, a PTZ analogue has shown interesting results during the in vivo studies of the full length h-TLK1B, when combined with Bicaltumide (BIC), known drug for prostate cancer ${ }^{24}$. We removed the $\mathrm{C} 2$ substitution and found that $\mathrm{J} 54$ had better inhibitory properties as compared to THD. Here, in the second generation TLK inhibitors, we decided to study the substitution at the R1, A, B and R2 position of the general scaffold (Fig. 4) to study the overall structure activity relationship of the molecule with hTLK1B-KD. All the compounds were synthesized at our facility and synthetic schemes of some of the inhibitors were previously reported ${ }^{36,37}$; rests are discussed here in the supplementary information. The inhibitory effects of the synthesized inhibitors were compared with Stuarosporine, an universal kinase inhibitor, taken as a positive control. 
Table 1

The phenothiazine analogues synthesized for the structureactivity relationship studies. The molecules were synthesized with substitutions at the R1, A or B, and R2 positions of the parent scaffold (Fig. 4).

\begin{tabular}{|c|c|c|c|c|c|}
\hline Compound & R1 & A & B & R2 & $x$ \\
\hline 1 & $S$ & $\mathrm{CO}$ & $\mathrm{CH}_{2}$ & Piperazine & $\mathrm{H}$ \\
\hline 2 & $S$ & $\mathrm{CO}$ & $\mathrm{CH}_{2}$ & Morpholine & $\mathrm{H}$ \\
\hline 3 & $S$ & $\mathrm{CH}_{2}$ & $\mathrm{CO}$ & Pyrolidine & $\mathrm{H}$ \\
\hline 4 & $S$ & $\mathrm{CH}_{2}$ & $\mathrm{CO}$ & Morpholine & $\mathrm{H}$ \\
\hline 5 & $\mathrm{SO}_{2}$ & $\mathrm{CH}_{2}$ & $\mathrm{CO}$ & Morpholine & $\mathrm{H}$ \\
\hline 6 & $S$ & $\mathrm{CH}_{2}$ & $\mathrm{CO}$ & Piperadine & $\mathrm{H}$ \\
\hline 7 & $\mathrm{SO}_{2}$ & $\mathrm{CH}_{2}$ & $\mathrm{CO}$ & Morpholine & $\mathrm{H}$ \\
\hline 8 & $\mathrm{SO}_{2}$ & $\mathrm{CH}_{2}$ & $\mathrm{CO}$ & Piperidine & $\mathrm{H}$ \\
\hline 9 & $S$ & $\mathrm{CO}$ & $\mathrm{CH}_{2}$ & Piperidine & $\mathrm{H}$ \\
\hline 10 & $S$ & $\mathrm{CO}$ & $\mathrm{CH}_{2}$ & Methyl piperizine & $\mathrm{H}$ \\
\hline 11 & $S$ & $\mathrm{CO}$ & $\mathrm{CH}_{2}$ & Pyrolidine & $\mathrm{H}$ \\
\hline 12 & $\mathrm{CO}$ & $\mathrm{CH}_{2}$ & $\mathrm{CH}_{2}$ & Morpholine & $\mathrm{H}$ \\
\hline 13 & $\mathrm{CO}$ & $\mathrm{CH}_{2}$ & $\mathrm{CH}_{2}$ & Piperidine & $\mathrm{H}$ \\
\hline 14 & $S$ & $\mathrm{CO}$ & $\mathrm{CH}_{2}$ & tetrazole & $\mathrm{H}$ \\
\hline 15 & $S$ & $\mathrm{CH}_{2}$ & $\mathrm{CH}_{2}$ & Pyrolidine & $\mathrm{H}$ \\
\hline 16 & $S$ & $\mathrm{CH}_{2}$ & $\mathrm{CH}_{2}$ & Amine & $\mathrm{H}$ \\
\hline 17 & $S$ & $\mathrm{CH}_{2}$ & $\mathrm{CH}_{2}$ & Piperidine & $\mathrm{H}$ \\
\hline 18 & $\mathrm{CO}$ & $\mathrm{CH}_{2}$ & $\mathrm{CO}$ & Morpholine & $\mathrm{H}$ \\
\hline 19 & $\mathrm{CO}$ & $\mathrm{CH}_{2}$ & $\mathrm{CO}$ & Piperidine & $\mathrm{H}$ \\
\hline
\end{tabular}

The in-vitro screening of the in-house synthesized inhibitors showed that the compounds $6[2-(10 \mathrm{H}-$ phenothiazin-10-yl)-1-(piperidin-1-yl)ethan-1-one] and 14 [1-(10H-phenothiazin-10-yl)-2-(1 H-tetrazol- 
1-yl)ethan-1-one] inhibited the hTLK1B-KD marginally higher as compared to THD in presence of different substrates of TLK1B; Nek1 and ASF1a respectively. As expected, the results from the in vitro biochemical analysis (Supplementary Figure S10) exactly corroborated with our anticipation. All our synthesized compounds were free of $\mathrm{C}_{2}$-substitutions but contained a two-carbon short alkyl chain connector between the phenothiazine ring and the terminal amine, which we expect to govern the reduced affinity for the D2 receptors (Table 1). Besides, we introduced a carbonyl group in the substituted side chain (alkyl chain linker, A, and B carbon) in the newly synthesized inhibitors, which participates in the intra-/inter-molecular hydrogen bond formation, thereby contributing to the enhanced

biological activity of the molecules ${ }^{38}$. We found that this carbonyl substitution either in the A or B position is improving the inhibition of the synthesized molecule than that of J54 or THD. Further, when the sulphur in the R1 position was substituted with either sulfonyl or carbonyl, the molecules are either not inhibiting $(5,8,12,13)$ or enhancing the activity of the TLK-KD (compound 18 and 19) (Supplementary Figure, S10). The structural modification on the sulphur group (S-dioxide), which increases the electronic effect and causes a change in its molecular structure within the binding sites, thereby decreasing its selectivity. We identified two second generation phenothiazine inhibitors 6 and 14 in the micromolar range $7.5 \mu \mathrm{M}$ and $6.1 \mu \mathrm{M}$ respectively (Fig. 5). These results are important as TLK1B is a very less explored drug target in the cancer therapeutics. This further confirms that our recombinantly purified TLK1B-KD can be a usable drug target for the screening of TLK inhibitors and can easily distinguish inhibitors in the presence of different substrates.

Nevertheless, further SAR analysis is required to investigate the role of different heterocyclic substitutions modifying the terminal amine motif in the designed molecules. We understand that our study is preliminary; however, the cell-based cytotoxicity, validation in the relevant pre-clinical cancer models, and in vivo xenograft studies of the compounds 6 and 14 are underway. Once validated, in the future, we plan to test these compound hits in combination with the androgen-deprivation therapy (ADT) to target TLK1 in prostate cancer to understand the inhibitor-protein interaction and their effect on the downstream $\operatorname{targets}^{24,39}$.

\section{Discussion}

The article outlines how to (i) construct a hTLK1B-KD-LPP-pETDUET-1 expression vector by the conventional Polymerase Chain Reaction (PCR)-based cloning approach; (ii) Soluble expression and purification of large scale biologically active target protein (iii) in-vitro drug screening of purified recombinant hTLK1B-KD with the in-house synthesized phenothiazine compounds. Even though we had purified the hTLK1B full length protein previously, the low catalytic efficiency of the kinase was a challenge during the screening of TLK inhibitors. Furthermore, the full length kinase contains a disordered region which reduces the crystallbilty of the protein. With the aim for a better inhibitor screening platform and for successful crystallization studies, we hence decided to truncate and express the major catalytic domain of the hTLK1B known as hTLK1B-Kinase domain. The PCR based cloning method helped us to amplify the kinase domain region of the protein along with the required restriction sites for directional 
cloning during the ligation. Often during the expression of the kinases, low solubility, incorrect folding, and heterogeneous autophosphorylation are significant bottlenecks particularly in Escherichia coli. Hence, the utilization of bacteriophage $\lambda$ protein phosphatase coexpression strategy has been rapidly gaining in popularity ${ }^{20}$. Alternatively, a lot of solubility-enhancing tags such as maltose-binding protein (MBP), NusA, and glutathione S-transferase (GST) are widely employed in molecular biology to avoid the formation of insoluble protein aggregates. However, proteolytic cleavage of these solubility enhancers is advised before conducting structural and functional studies as they may affect the protein properties and function due to their large sizes ${ }^{56}$. The $6 x$-polyhistidine tag, on the contrary, overcomes these limitations. The relatively small size and charge of the $6 x$-polyhistidine tag sporadically affect the protein function, thus, preserving the integrity of the polyhistidine fusion proteins ${ }^{40}$. Once the clones with the correct insert are identified, they are ready for the downstream applications and further expression in bacterial system.The expression of recombinant hTLK1B-KD at $25^{\circ} \mathrm{C}$ for 9 hours with $0.5 \mathrm{mM}$ IPTG yielded a $50 \%$ soluble fraction of the protein. The recombinant hTLK1B-KD protein used here exemplifies the most desirable outcome based on the generic IMAC purification process. Generally, after the first IMAC step, the purity of the fusion protein is typically in the range of $70-80 \%$ as endogenous proteins bind nonspecifically to the resin. Hence, the second round of IMAC is required to improve the purity of the protein. However, in the case of hTLK1B-KD, a significant improvement in purity and yield is observed just after the single affinity purification step. Our one-step purification strategy yields about $\sim 10 \mathrm{mg}$ of the pure, stable, homogeneously unphosphorylated, well-folded and biologically active hTLK1B-KD protein per litre of the bacterial culture.

Kinases have become a proven target class for the new anticancer drugs, and the goal is to identify more of these that could reshape the available therapeutic options. The TLKs are becoming the centre of much attention for their role in DSB repair ${ }^{41-43}$, and their potential contribution to cancers refractory to XRT or $\mathrm{RMT}$, including cholangiocarcinoma ${ }^{44}, \mathrm{BCA}^{45}$, and $\mathrm{PCa}^{43}$. Since the mechanism of action of TLKs in cancer, chemoresistance is now relatively well elucidated ${ }^{21}$, adding the TLK-specific inhibitors could significantly improve the outcome. The PTZs antipsychotics used for the treatment of schizophrenia were identified as the first Human TLK inhibitors, but with the potential side-effects ${ }^{46}$. We understood this possible side effect arised from the $\mathrm{C} 2$ substitution of the PTZs, hence we contemplated that the substitution/removal of the $\mathrm{C} 2$ electron-withdrawing groups would affect the overall orientation and reactivity of the molecule, thereby decreasing the binding affinity towards the dopamine receptor and lowering its antipsychotic effect ${ }^{47}$. As reported, the extent of the significant tranquilizer impact of phenothiazine's will be reduced as within the order of piperazine < piperidine < alkyl chain; supported the pharmacological exploitation of the various substituents within the terminal amine substituent ${ }^{37}$. From a drug-design perspective, morpholine, piperidine, and piperazine substructures are highly privileged molecules ${ }^{34,35}$. So, we have fixed two alkyls chain linkers and chosen morpholine, piperidine, piperazine, methyl piperazine, tetrazole and pyrolidine, moiety as terminal groups attributable to their wide range of biological properties ${ }^{38}$. Furthermore, it was stated that the substitution of a carbonyl group, irrespective of the position, whether in the phenothiazine or the side-chain substituent, contributes to its 
pharmacological properties ${ }^{48}$. Keeping this in mind, we explored the PTZs at the N10 (H), R1, A and B positions while removed the substitution at $\mathrm{C} 2$, along with customizing the alkyl chain connector length ${ }^{49}$ to design, synthesize and develop a library of next-generation TLK1 inhibitors, after THD, to circumvent the adverse effects of the PTZs and advance them in the clinic. From the screening with the purified hTLK1B-KD, we identified two potential molecules, 6 and 14 that showed significant inhibition with the hTLK-KD. However, further cell-based and in-vivo validation is required, which is yet underway. We expect that our identified compounds would effectively inhibit the TLK1/NEK1 axis in prostate cancer when tested in combinatorial appraoch with the androgen-deprivation therapy (ADT), the current standard method of treatment for the advanced Prostate Cancer (PCa). The hTLK1B-KD is a potential biomarker and a drug target for prostate cancer and further studies on hTLK1B will put light on its role in many crucial pathways.

\section{Methods}

\subsection{Enzymes and chemicals}

All restriction endonucleases and DNA polymerase enzymes were procured from New England Biolabs (Ipswich, MA, USA). Oligonucleotides for PCR-based cloning were obtained from Integrated DNA Technologies (IDT), Inc. (Illinois, USA). Escherichia coli DH5a ${ }^{\text {TM }}$ and Rosetta Gami $2^{\text {TM }}$ (DE3) pLysS were purchased from Novagen (Merck Biosciences Division, Darmstadt, Germany), DNA extraction/purification kits were from Qiagen (Hilden, Germany), Nuvia ${ }^{\text {TM }}$ Immobilized Metal Affinity Chromatography (IMAC) Resin charged with $\mathrm{Ni}^{2+}$ was obtained from Bio-Rad Laboratories (Hercules, CA, USA). HiLoad 16/600 Superdex 75 pg gel filtration chromatography column was purchased from GE Healthcare (Chicago, Illinois, USA). ADP-GIo ${ }^{\text {TM }}$ Kinase Assay Kit was obtained from Promega Corporation (Madison, WI, USA). Complete $^{\text {TM }}$ EDTA-Free Protease Inhibitor tablets were obtained from Roche (Basel, Switzerland). All other molecular biology reagents were from Sigma-Aldrich (Darmstadt, Germany).

\subsection{Vector designing and cloning}

The hTLK1B_KD-LPP-pETDUET-1 expression construct was made as described previously ${ }^{20}$ containing a bacteriophage $\lambda$ phosphatase ORF in the MCS-2 of the pETDUET-1 vector for soluble and dephosphorylated expression of the target protein. The 919 bp hTLK1B-Kinase Domain (hTLK1B_KD, amino acids 230-530, UniProt- Q9UKI8-3) gene was PCR amplified by forwarding 30-mer (pETDUETKD_FOR, 5'-ACAGAGGATCCAAAGGACCACCCGACCCTG-3'), bearing a BamHI restriction site (underlined) and reverse 38-mer (pETDUET-KD_REV, 5'-TCGTTGTCGTCGCCATTGGACAAGATCTTAAGACGACA-3'), bearing an EcoRI restriction site (underlined). We inserted the purified PCR product, now bearing the appropriate restriction enzyme overhangs into the MCS-1 (BamHI/EcoRI) of the vector pETDUET-1 with the 6x-polyhistidine tag at the N-terminus. Cloning of hTLK1B_KD ORF (in MCS-1) and subcloning of LPP ORF (in MCS-2) into this vector containing a T7 promoter/lac operator allows T7 RNA polymerasedependent coexpression of hTLK1B_KD and LPP at the same time. The constructed plasmid was validated by restriction digestion analysis and direct sequencing. 


\subsection{Protein expression and purification}

The hTLK1B_KD-LPP-pETDUET-1 was chemically transformed in E. coli Rosetta Gami $2^{\text {TM }}$ (DE3) pLysS, inoculated in Luria-Bertani broth with $100 \mu \mathrm{g} / \mathrm{ml}$ ampicillin as a antibiotic marker gene at $37^{\circ} \mathrm{C}$. The media was induced with $0.5 \mathrm{mM}$ IPTG once the $0^{. D_{600}}$ was reached to $0.6-0.8$. The further incubation was done at a reduced temperature to $25^{\circ} \mathrm{C}$ for protein expression. After 9 hours, the cells were harvested by centrifugation (10 min for $4500 \mathrm{~g}$ ) and the supernatant was discarded. The cell pellet can be stored at $-80^{\circ} \mathrm{C}$ until further use. For the protein purification, the cell pellet was resuspended in the lysis buffer containing $50 \mathrm{mM}$ Tris-Cl (pH-8.0), $300 \mathrm{mM} \mathrm{NaCl}, 10 \%$ Glycerol, 1 mM PMSF, 1\% Triton-X 100, $5 \mathrm{mM}$ imidazole, and $0.3 \mathrm{mM}$ TCEP. A tablet of Complete EDTA-free protease inhibitor cocktail was added to the resuspended cell lysate, and the lysis was completed through probe sonication at amplitude of $45 \%$ (10s ON/20s OFF) while cooling on ice for 10 minutes. The lysate was centrifuged at $20,000 \mathrm{~g}$ for 45 mins at $4^{\circ} \mathrm{C}$, and the pellet was discarded. The lystae was loaded to a column containing 2-ml Ni-NTA resin, preequilibrated with $50 \mathrm{mM}$ Tris-Cl (pH-8.0), $300 \mathrm{mM} \mathrm{NaCl}, 20 \mathrm{mM}$ imidazole, and $0.3 \mathrm{mM}$ TCEP. After running the lysate through, the non-specific proteins were washed off with $50 \mathrm{mM}$ Tris- $\mathrm{Cl}(\mathrm{pH}-8.0), 300$ $\mathrm{mM} \mathrm{NaCl}$, and $0.3 \mathrm{mM}$ TCEP containing $50 \mathrm{mM}$ imidazole. The protein was eluted with $50 \mathrm{mM}$ Tris-Cl $(\mathrm{pH}-8.0), 450 \mathrm{mM} \mathrm{NaCl}, 5 \%$ Glycerol, and $0.3 \mathrm{mM}$ TCEP containing $200 \mathrm{mM}$ imidazole. The eluted protein was concentrated and loaded onto HiLoad 16/60 Superdex 75 column for buffer-exchange with $50 \mathrm{mM}$ Tris-Cl, $50 \mathrm{mM} \mathrm{NaCl}, 0.2 \mathrm{mM}$ TCEP and as a final polishing step. 12\% SDS-PAGE was used to monitor the efficiency of the purification steps. The pure fractions were pooled together, concentrated using a centrifugal concentrator ( $10 \mathrm{kDa}$ Amicon filter). The concentration of the protein was estimated by absorbance method (Bradford assay) at $280 \mathrm{~nm}$ as well as using the calculated extinction coefficient of $43,320 \mathrm{M}^{-1} \mathrm{~cm}^{-1}$.

\subsection{Circular Dichroism}

The CD spectrum was recorded in a $0.5 \mathrm{~mm}$ quartz cuvette at $25^{\circ} \mathrm{C}$ with a scanning speed of $0.5 \mathrm{~nm} / \mathrm{s}$ and a bandwidth of $0.5 \mathrm{~nm}$ using J-815 circular dichroism (CD) spectropolarimeter (Jasco, Inc., MD, USA). The purified recombinant 6x-polyhistidine-tagged hTLK1B_KD protein ( $99 \%$ pure) was prepared in $50 \mathrm{mM}$ Tris-Cl, $50 \mathrm{mM} \mathrm{NaCl}$ and $0.3 \mathrm{mM} \mathrm{TCEP}(\mathrm{pH}-8.0)$ at a final concentration of $0.5 \mathrm{mg} / \mathrm{ml}$. The protein sample was filtered through $0.22 \mu \mathrm{m}$ Millex-GP filters and was ensured free of the particulate matter.The data is calculated from the mean of three replicative sets of experiments and the curve was fitted using GraphPad Prism software (Version 6.01, La Jolla, CA). The baseline data was subtracted from each data set using a buffer control. The CD scans were recorded, and the intensity of the signal was maintained below the transmission limit of the instrument.

\subsection{ADP-GLo Kinase assay}

The ADP-Glo kinase assay was performed in a solid, white, flat bottom 96-well plate in a reaction volume of $25 \mu$ with $2 X 1 \mu \mathrm{M}$ kinase (e.g., hTLK1B_KD), 2X $1 \mu \mathrm{M}$ kinase substrate (e.g., ASF1a/ Nek1/ Histone $\mathrm{H} 3), 2 \mathrm{X} 100 \mu \mathrm{M}$ ATP in 1X Kinase Reaction Buffer (KRB). The 1X KRB comprised of $40 \mathrm{mM}$ Tris- $\mathrm{Cl}(\mathrm{pH}-$ 
7.5), $20 \mathrm{mM} \mathrm{MgCl}$ and $0.1 \mathrm{mg} / \mathrm{ml}$ Bovine Serum Albumin (BSA). The reaction was initiated immediately upon addition of ATP and incubated for 30 minutes at room temperature to complete the reaction. After 45 minutes, the enzymatic reaction was terminated by quenching the excess ATP using $25 \mu \mathrm{L}$ of ADPGLo reagent. In the end, the ADP in the reaction is converted to ATP followed by luciferase reaction to emit light by adding $50 \mu$ of a Kinase Detection Reagent to each well and incubated for 60 minutes in dark. For the preliminary inhibitor sensitivity measurements, $2 \mu$ inhibitor was added at a final concentration of $20 \mu \mathrm{M}$ into the $25 \mu \mathrm{L}$ volume of kinase reaction for the preliminary screening of inhibitors (<5\% DMSO). DMSO was used as a suitable control vehicle. During the dose response, $2 \mu \mathrm{L}$ from serially diluted concentrations of inhibitor from $160 \mu \mathrm{M}$ to $0.1560 \mu \mathrm{M}$ was added to the enzyme reaction. The generated luminescent signal was readout using GraphPad Prism 6 (GraphPad Software Inc., CA, USA), and the data curve was fitted by non-linear regression model to get a best-fit value that applies to all data sets.

\section{Declarations}

\section{Acknowledgment}

This work was financially supported by DRDO and SERB. The authors thank Indian Institute of Technology, Gandhinagar for the fellowship and infrastructure. S.K. greatly acknowledges Kankuben Bakshirambhai Gelot Chair for the support.

\section{Author contributions}

S.K. and V.T. conceptualized the idea and designed the protocol along with S.B. and J.H. D.J. carried out the major experiments as well as compiled the manuscript. J.H., P.S., T.H. synthesized the molecules. S.B. and V.C. performed the cloning and expression experiments.

\section{Competing interest}

The authors declare no competing financial interests

\section{Data Availability}

UniProt ID of TLK1B: Q9UKI8

\section{References}

1. Cormier, K. W., \&Woodgett, J. R. Protein Kinases: Physiological Roles in Cell Signalling. eLS, 1-9.

2. Knight, Z. A., Lin, H. \&Shokat, K. M. Targeting the cancer kinome through polypharmacology. Nat. Rev. 10, 130-137 (2010).

3. Müller, S., Chaikuad, A., Gray, N. S. \& Knapp, S. The ins and outs of selective kinase inhibitor development. Nat. Chem. Biol,11, 818-821 (2015). 
4. Siegel, R. L., Miller, K. D., Fuchs, H. E. \&Jemal, A. Cancer Statistics, 2021. CA Cancer J Clin. 71, 7-33 (2021).

5. Stegmeier, F., Warmuth, M., Sellers, W.\&Dorsch, M. Targeted cancer therapies in the twenty-first century: lessons from imatinib. Clin. Pharmacol. Ther.87, 543-552 (2010).

6. Bhullar, K. S.et al. Kinase-targeted cancer therapies: progress, challenges and future directions. Mol. Cancer. 17, 1-20 (2018).

7. Knapp, S. \&Sundström, M. Recently targeted kinases and their inhibitors-The path to clinical trials. CurrOpinPharmacol.17, 58-63 (2014).

8. Wu, P., Nielsen, T. E.,\& Clausen, M. H. FDA-approved small-molecule kinase inhibitors. Trends Pharmacol.Sci.36, 422-43 (2015).

9. Volkamer, A., Eid, S., Turk, S., Jaeger, S.,Rippmann, F. \&Fulle, S.Pocketome of human kinases: prioritizing the ATP binding sites of (yet) untapped protein kinases for drug discovery. J Chem/nf Model.55, 538-549 (2015).

10. Noble, M. E., Endicott, J. A. \& Johnson, L. N. Protein kinase inhibitors: insights into drug design from structure. Science. 303, 1800-1805 (2004).

11. Johnson, L. N.; Noble, M. E. \&Owen, D. J., Active and inactive protein kinases: structural basis for regulation. Cell.85, 149-158 (1996).

12. Karaman, M. W. et al. A quantitative analysis of kinase inhibitor selectivity. Nat.Biotechnol.26, 127132 (2008).

13. Médard, G.et al. Optimized chemical proteomics assay for kinase inhibitor profiling. J.Proteome Res.14, 1574-1586 (2015).

14. Davis, M. I. et al. Comprehensive analysis of kinase inhibitor selectivity. Nat. Biotechnol.29, 10461051 (2011).

15. Jaeger, J. Macromolecular Structure Determination by X-ray Crystallography.eLS. 2001.

16. Chambers, S. P., Austen, D. A., Fulghum, J. R.\& Kim, W. M. Purification, High-throughput screening for soluble recombinant expressed kinases in Escherichia coli and insect cells. Protein Expr. Purif. 36, 4047 (2004).

17. Wang, L. et al. High yield expression of non-phosphorylated protein tyrosine kinases in insect cells.Protein Expr.Purif. 61, 204-211 (2008).

18. Seeliger, M. A. et al. High yield bacterial expression of active c-Abl and c-Src tyrosine kinases. Protein Sci.14, 3135-3139 (2005).

19. Albanese, S. K. et al.An open library of human kinase domain constructs for automated bacterial expression. Biochemistry.57, 4675-4689 (2018).

20. Bhoir, S., Shaik, A.,Thiruvenkatam, V.\&Kirubakaran, S. High yield bacterial expression, purification and characterisation of bioactive Human Tousled-like Kinase 1B involved in cancer. Sci.Rep.8, 1-9 (2018).

21. De Benedetti, A. The tousled-like kinases as guardians of genome integrity. Int. Sch. Res. Notices. 2012 (2012). 
22. Parton, D. L. et al. An open library of human kinase domain constructs for automated bacterial expression. bioRxiv. 038711 (2016).

23. Garrote, A. M., Redondo, P., Montoya, G.\& Muñoz, I. G. Purification, crystallization and preliminary Xray diffraction analysis of the kinase domain of human tousled-like kinase 2. ActaCryst. F.70, 354357 (2014).

24. Singh, V. et al. Generation of phenothiazine with potent anti-TLK1 activity for prostate cancer therapy. Iscience. 23, 101474 (2020).

25. Chong, H.\& Guan, K. L. Regulation of Raf through phosphorylation and N terminus-C terminus interaction. J.Biol. Chem.278, 36269-36276 (2003).

26. Sillje, H., Takahashi, K., Tanaka, K., Van Houwe, G.\&Nigg, E. Mammalian homologues of the plant Tousled gene code for cell-cycle-regulated kinases with maximal activities linked to ongoing DNA replication. EMBO J.18, 5691-5702 (1999).

27. Kristiansen, J. E.\& Mortensen, I.Antibacterial effect of four phenothiazines. Pharmacol.Toxicol. 60, 100-103 (1987).

28. Domínguez, J. N. et al. Synthesis and antimalarial effects of phenothiazine inhibitors of a Plasmodium falciparum cysteine protease. J.Med. Chem.40, 2726-2732 (1997).

29. Swales, W. Tests of phenothiazine as anthelmintic: on a means of administration and the indicated uses for the control of parasitic diseases of sheep. Can JComp Med. 3, 188 (1939).

30. Lasagna, L.\&DeKornfeld, T. J.Methotrimeprazine: a new phenothiazine derivative with analgesic properties. JAMA. 178, 887-890 (1961).

31. Saggiomo, A. J.\& Sutton, B. M. Salicylic acid analogs of phenothiazine as antiinflammatory agents. J.Med. Chem.11, 1089-1090 (1968).

32. Ford, J. M., Prozialeck, W. C.\&Hait, W. Structural features determining activity of phenothiazines and related drugs for inhibition of cell growth and reversal of multidrug resistance. Mol.Pharmacol.35, 105-115 (1989).

33. Ronald, S. et al. Phenothiazine inhibitors of TLKs affect double-strand break repair and DNA damage response recovery and potentiate tumor killing with radiomimetic therapy. Genes cancer.4, 39-53 (2013).

34. Matthews, N., Franklin, R. J.\& Kendrick, D. A. Structure-activity relationships of phenothiazines in inhibiting lymphocyte motility as determined by a novel flow cytometric assay.

Biochem.Pharmacol.,50, 1053-1061 (1995).

35. Tapias, V., McCoy, J. L.\&Greenamyre, J. T. Phenothiazine normalizes the NADH/NAD+ ratio, maintains mitochondrial integrity and protects the nigrostriatal dopamine system in a chronic rotenone model of Parkinson's disease. Redox. Biol.24, 101164 (2019).

36. Hussain, J. et al. Synthesis and characterization of a new class of phenothiazine molecules with $10 \mathrm{H}$-substituted morpholine\&piperidine derivatives: a structural insight. J.Mol. Struct.1219, 128546 (2020). 
37. Hussain, J., Sahasrawat, P., Dubey, P., Kirubakaran, S. \&Thiruvenkatam, V.Exploring packing features of $\mathrm{N}$-substituted acridone derivatives: Synthesis and X-ray crystallography studies.

J. Mol. Struct.1248, 131448 (2021)

38. Jaszczyszyn, A. et al. Chemical structure of phenothiazines and their biological activity. Pharmacol Rep.64, 16-23 (2012).

39. Singh, V., Jaiswal, P. K., Ghosh, I., Koul, H. K., Yu, X. \& De Benedetti, A. Targeting the TLK1/NEK1 DDR axis with Thioridazine suppresses outgrowth of androgen independent prostate tumors. Int $J$ Cancer.145, 1055-1067 (2019).

40. Kimple, M. E., Brill, A. L. \& Pasker, R. L. Overview of Affinity Tags for Protein Purification. Curr. Protoc. Protein Sci.9, 608-616 (2013).

41. Sunavala-Dossabhoy, G. \& De Benedetti, A. Tousled Homolog, TLK1, Binds and Phosphorylates Rad9; TLK1 Acts as a Molecular Chaperone in DNA Repair. DNA Repair (Amst).8, 87-102 (2009).

42. Stevens, K. N. et al. Evaluation of Associations between Common Variation in Mitotic Regulatory Pathways and Risk of Overall and High Grade Breast Cancer. Breast Cancer Res. Treat.129, 617-622 (2011).

43. Ronald, S., Sunavala-Dossabhoy, G., Adams, L., Williams, B. \& De Benedetti, A. The Expression of Tousled Kinases in CaP Cell Lines and Its Relation to Radiation Response and DSB Repair. Prostate.71, 1367-1373 (2011).

44. Takayama, Y. et al. Silencing of Tousled-like Kinase 1 Sensitizes Cholangiocarcinoma Cells to Cisplatin-Induced Apoptosis. Cancer Lett.296, 27-34 (2010).

45. Byrnes, K. W. et al. Correlation of TLK1B in Elevation and Recurrence in Doxorubicin-Treated Breast Cancer Patients with High EIF4E Overexpression. J. Am. Coll. Surg.204, 925-933 (2007).

46. Centre, W. H. O. C.; Drug, I.; No, M. Who Pharmaceuticals. Qual. Assur. No. 1, 1-27 (2004).

47. Vitaku, E., Smith, D. T. \& Njardarson, J. T. Analysis of the Structural Diversity, Substitution Patterns, and Frequency of Nitrogen Heterocycles among U.S. FDA Approved Pharmaceuticals. J. Med. Chem.57, 10257-10274 (2014).

48. Pal'chikov, V. A. Morpholines. Synthesis and Biological Activity. Russ. J. Org. Chem.49, 787-814 (2013).

49. Patil, P., Madhavachary, R., Kurpiewska, K., Kalinowska-Tłuścik, J. \& Dömling, A. De Novo Assembly of Highly Substituted Morpholines and Piperazines. Org. Lett.19, 642-645 (2017).

\section{Figures}


a

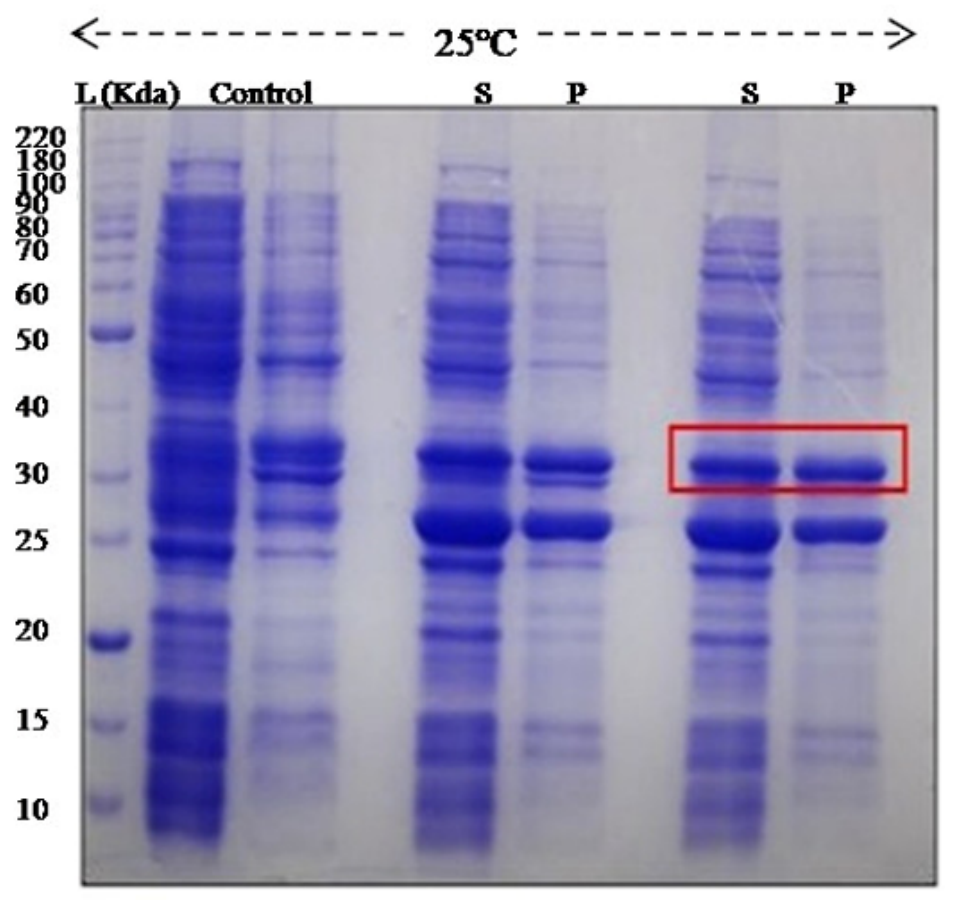

b

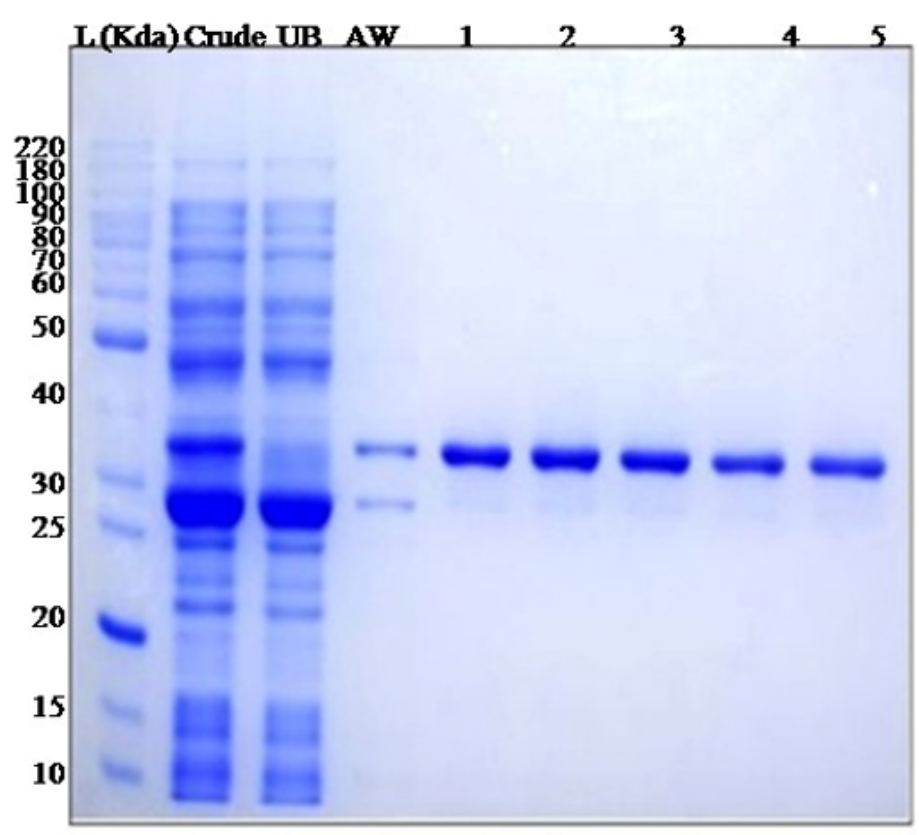

\section{Figure 1}

Expression and purification of hTLK1B-KD. (a) Coexpression of recombinant 6x-polyhistidine-tagged hTLK1B-KD along with bacteriophage $\lambda$ protein phosphatase at $25 \circ \mathrm{C}$ induced at different IPTG concentrations $(0.3 \mathrm{mM}$ and $0.5 \mathrm{mM})$. Control sample was taken as the uninduced. (b) Purification of recombinant $6 \mathrm{x}$-polyhistidine-tagged hTLK1B-KD after Ni-NTA purification steps. The abbreviations are as follows: L-molecular weight marker in kDa; S-supernatant; P-pellet, Crude-IPTG induced soluble fraction (total cell lysate-before IMAC); UB-unbound protein fraction; AW-after-binding column wash fractions; lanes 1-5, eluted hTLK1B-KD (36.6 kDa) after $200 \mathrm{mM}$ imidazole wash.
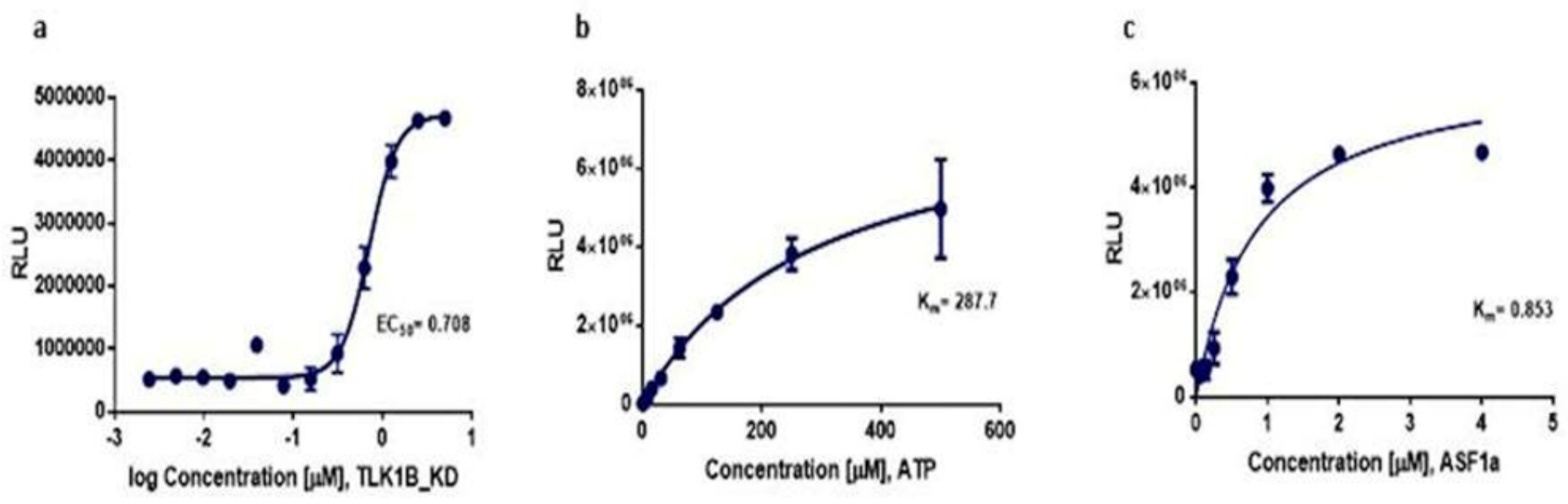

Figure 2 
Kinetic parameter of the purified hTLK1B-KD. (a) Assessing the bioactivity of hTLK1B-KD using ADP-Glo ${ }^{\text {TM }}$ Kinase Assay. The biological activity and optimal amount (EC50) of hTLK1B-KD kinase were determined by a twofold serial dilution in 1X Kinase Reaction Buffer using the desired amount of ATP (up to $1 \mathrm{mM}$, from b) and kinase substrate (from c). (b) and (c) Determination of the Km for ATP and Km for the substrate. The serial, twofold dilutions of ATP ( $f \circ r$ ) and kinase substrate (for $c$ ) were made respectively across the plate using $1 \mathrm{X}$ Kinase Reaction Buffer. The desired amount of kinase was utilized as determined in (a). The abbreviations are as follows: ADP, adenosine diphosphate; ATP, adenosine triphosphate; ASF1a, anti-silencing factor 1a.

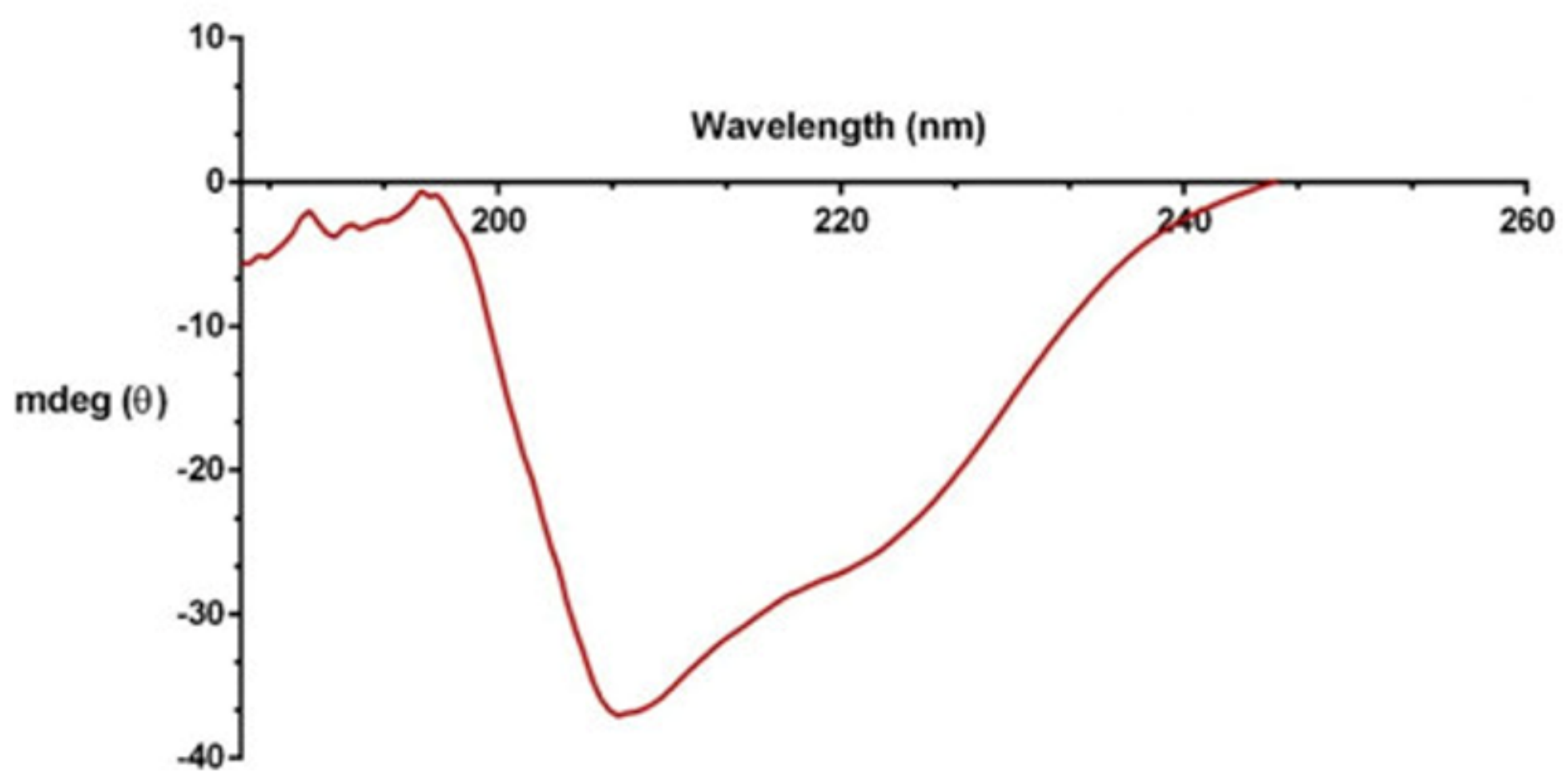

\section{Figure 3}

Secondary structure determination of hTLK1B-KD by circular dichroism (CD). The data demonstrates the presence of alpha-helices (73.68\%) and beta sheets (1.21\%) in the secondary structure of hTLK1B-KD 
Phenothiazine ring

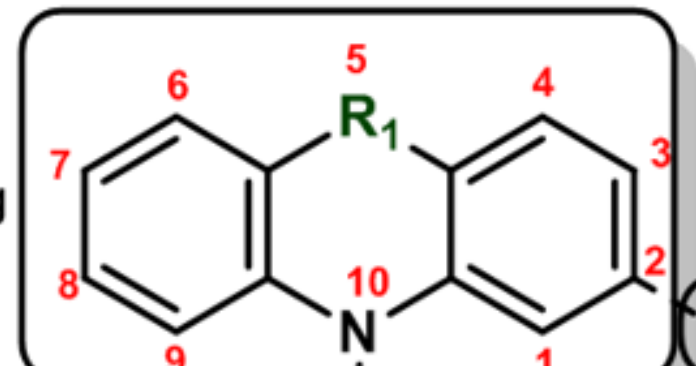

(X) c2- substituent

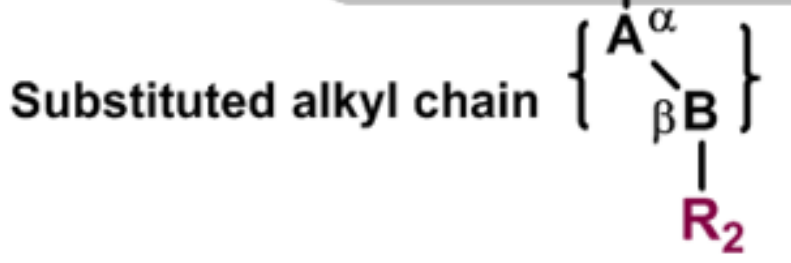

Terminal Amine substituent

Figure 4

General scaffold of the synthesized phenothiazine analogues. The major substitutions were made at the $R 1, A, B$, and R2 positions of the scaffold for the SAR studies.
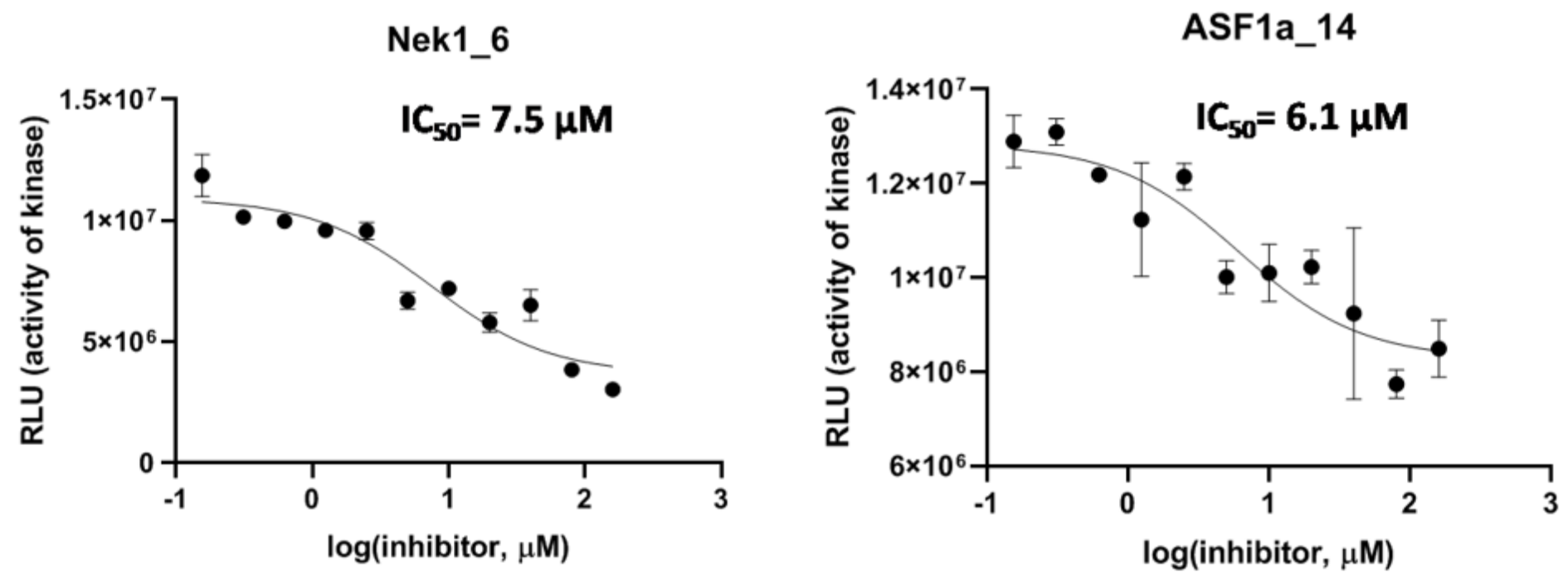

Figure 5

The dose response curve of 6 and 14 with TLK1B-KD. The two inhibitors 6 and 14 showed significant inhibition against TLK1B-KD in presence of Nek1 and ASF1a, when taken as substrates.

\section{Supplementary Files}

This is a list of supplementary files associated with this preprint. Click to download. 
- TLK1BKDSupplementaryinfo050222.docx

Page 19/19 